denum aspirated at I $_{5}$ minute intervals for one hour. Each sample is centrifugalised and the deposit examined microscopically. Crystals of cholesterin, pus cells or red blood cells indicate that the mucous membranes of the gall-bladder is unhealthy.

\title{
The Liver Function Tests.
}

It may be necessary to get some indication from special tests as to whether the function of the liver substance itself is normal or abnormal; there are several tests that can be used.

I. The icteric index.-Serum or plasma diluted if necessary with saline is matched against an arbitrary standard of potassium bichromate. Normal blood gives 4-6 units; up to I5 units is latent jaundice, and above $\mathrm{I}_{5}$ units there is visible jaundice.

2. The laevulose test.- 50 grms. of laevulose are used for the test given in the same way as glucose for the glucose tolerance test. Laevulose has no effect on the blood sugar in normal persons. When, however, there is liver insufficiency a rise occurs resembling the normal blood sugar curve after glucose ingestion. The duration and height of the rise is proportionate, roughly to the degree of hepatic insufficiency.

3. The hippuric acid test.-The bladder is emptied between two meals and the patient is then given 4 grms. of sodium benzoate in $200 \mathrm{cc}$. of water flavoured with peppermint; he empties his bladder one, two, and three hours after, and the whole of the specimens are saved for estimation of the hippuric acid. No food should be taken during the test, but water is allowed should he desire it. Benzoic acid is converted in transit through the liver into hippuric acid, and the result is expressed as the percentage of sodium benzoate ingested. Normally 80 to Ioo per cent of the hippuric acid will be found in the urine within 4 hours. In abnormal cases of liver disease the excretion is much slower.

4. The sucrose test.-No food is taken after 8 p.m. The next morning at 9 a.m. 20 cc. of blood are collected and the patient is then given a drink of Ioo grms. of sucrose (Cane sugar) in $300 \mathrm{cc}$. of water flavoured with tincture of orange. Further samples of blood (about $\mathrm{I} \cdot 5 \mathrm{cc}$.) are taken at 30,60, 90, I20 and I50 minutes later, and the urine is collected before and 90 minutes after the drink. The amount of glucose and laevulose is estimated in each sample of blood, and the tests for the same are applied to the urine passed. In normal adults the blood glucose rises quickly to a maximum of I60 mgm. in about half an hour, and then falls slowly to the end of $2 \frac{1}{2}$ hours. The blood laevulose does not rise so quickly (to about Io mgm. in $I \frac{1}{2}$ hours), and then is sometimes a delayed fall, but in all cases the values fall to under $6 \mathrm{mgm}$. at the end of the $2 \frac{1}{2}$-hours period.

The writer would like to acknowledge the great help he has received in the biochemical details from Mr. G. A. Higgins, B.Sc., of the Biochemical Laboratory of the Radcliffe Infirmary; the details of the sucrose test and other of the data were the subject of a paper by him and Mr. J. R. P. O'Brien, B.Sc., an account of which was read at a meeting of the Physiological Societyat Manchester in July 1942, and which is now in course of preparation for publication.

\section{THE SURGERY OF GALL BLADDER DISEASE}

\author{
By H. E. GRIFFITHS, M.S., F.R.C.S. \\ (Surgeon to the Albert Dock Seamen's Hospital, etc.)
}

I.ong ago a lecturer told a class of medical students that the three most important qualifications of a successful surgeon were "Judgment, Judgment and Judgment."

To no sphere of surgery is this axiom more applicable than to that of the treatment of the gall bladder.

The edifice of surgical judgment is based on the solid foundation of exact anatomical knowledge; its walls are laboriously built with the bricks of experience and crowned with the dome of understanding. 
As the surgery of the gall bladder is too vast a subject to be covered in a single article no attempt will be made here to give a detailed study of the subject. On the contrary, only a few bricks from the experience of one surgeon will be offered younger surgeons in the hope that they may assist them in the building of their surgical judgment.

STONES FROM THE FOUNDATION

\section{SURGICAL ANATOMY}

An intimate knowledge of the normal anatomy of the gall bladder, its connections and surroundings, is an implied qualification of every man when he first enters this field of surgery.

Variations from the normal-or perhaps it would be better to say from the usual-are so frequently encountered that some of them may be enumerated here.

\section{The Gall Bladder.}

Whereas the gall bladder normally lies in its fossa on the under surface of the liver with its fundus completely clothed in peritoneum and its upper surface bare of peritoneum, the gall bladder may be found in any stage from being completely buried in the substance of the liver to suspension in a complete mesentery. When the mesentery is complete tortion of its pedicle may occur. Two such cases which I removed are in the College of Surgeons Museum, and both are interesting because the gall bladder was found lying with its long axis extending transversely from right to left, and in each. case-both very thin women-a sausage-like swelling could be felt lying across the epigastrium.

Variations in shape of the gall bladder are usually acquired, and the commonest is the hourglass gall bladder frequently containing a large stone in the proximal portion and a collection of small stones in the distal part. The danger of removing only the distal part of the hourglass is avoided by the routine identification of the T-junction of the cystic, common hepatic, and common bile ducts before ligature of the cystic duct.

Multiple gall bladders occur, but are extremely rare. The following is a description of the only case which I have seen. The diagnosis of gall stones had been made by the physician. The diagnosis was confirmed by X-ray, which showed clearly several moderate sized stones tightly packed together. The case was referred to me for operation, and on opening the abdomen the gall bladder in the usual site was felt to contain one small stone in the neck. Nothing else was seen, and I removed the gall bladder. I could not find any immediate explanation of the $\mathrm{X}$-ray shadows, and made a careful search of the right lobe of the liver. On its under surface, lateral to the fossa of the gall bladder, was a slight bulge, and palpation of this enabled me to feel what I suspected to be a stone. I incised the liver and found a spherical gall bladder packed with stones. The gall bladder shelled out quite easily, and I ligatured the duct leading into the substance of the liver in a direction suggesting that it joined the right hepatic duct in the fissure; but I did not think it right to explore further. No cystic artery was found. The patient made an uneventful recovery so that further dissection must be postponed.

\section{The Cystic Duct.}

The cystic duct varies considerably in length. Whereas the normal cystic duct has an S-shaped bend, the duct is occasionally so short as to run a straight course into the common bile duct. In other cases it runs for an inch or more parallel to the common hepatic duct before their junction forms the common bile duct, which of course is then much shorter than usual. The cystic duct occasionally enters the right hepatic duct. Accessory cystic ducts enter the gall bladder from the right lobe of the liver, usually in the bare area. Different writers have given different estimates of the frequency of their occurrence at from 5 to 25 per cent of cases. My personal experience, both as an anatomist and as a surgeon, has been that they are exceedingly rare in such size as to be noticeable, or to affect cholecystectomy whether during the operation or during the post-operative stage.

\section{The Right Hepatic Duct.}

A fairly common variation of the right hepatic duct is to find it making a loop up behind 
the gall bladder, sometimes entering the fossa for the gall bladder. In this position it is very liable to injury.

\section{The Common Bile Duct.}

The common bile duct is expected to lie in the free border of the gastro-hepatic omentum and to run a fairly straight course downwards and backwards to reach the duodenum. In its course it lies anterior to the portal vein. On two occasions I have seen and carefully demionstrated the portal vein lying in front of the common bile duct at the level of the entrance of the cystic duct.

\section{The Cystic Artery.}

The cystic artery commonly arises from the right hepatic artery and lies behind the cystic duct. It reaches the gall bladder at the origin of the cystic duct, and there divides into two lateral branches. The variations of origin of the cystic artery and its subsequent course are legion: it may arise direct from the aorta, or even from the right phrenic artery, to mention only two of the many. It behoves the surgeon, therefore, so to conduct his operation that he is ready to deal with the artery when he finds it and also to be on his guard against ligaturing the all-important right hepatic artery.

\section{Lymph Glands.}

Normally one gland is seen in the first bend of the $\mathrm{S}$ of the cystic duct. Occasionally lymph nodes are found along the cystic duct and extending along the course of the common bile duct to the head of the pancreas. More often, if any glands are found other than the "cystic gland" they are felt only at the upper border of the head of the pancreas, where they have not infrequently been mistaken for stones in the common bile duct.

\section{BRICKS FOR THE WALLS}

\section{OPERATIONS UPON THE GALL BLADDER}

Operations upon the gall bladder fall into two groups: those which are undertaken primarily for the relief of symptoms which are referable to the gall bladder itself and those which are undertaken for more remote causes.

In the first group are included cases of cholelithiasis, cholecystitis, both acute and chronic, carcinoma of the gall bladder, tortion (or volvulus) of the gall bladder, direct injuries to the gall bladder, and such freak cases as strangulation of the gall bladder in a femoral hernial sac.

In the second group will be included operations for the relief of obstructive jaundice from such causes as carcinoma of the head of the pancreas or of the common bile duct, stricture of the common bile duct, sclerosing pancreatitis, and operations in which the gall bladder is the site of infection, although not the seat of symptoms: acute and chronic pancreatitis, fibrositis and polyarthritis; typhoid and dysentry carriers, etc.

\section{General Considerations for Operations on the Gall Bladder}

The operations call for speed without haste; for a minimum amount of handling and of traction. For these reasons the plan of operation must be made before the abdomen is opened the surgeon being always prepared, like a good general; to modify his plan to meet the opposition which may be encountered. There must be no time lost in "dithering" at the unexpected. Two assistants should always be available, the first standing opposite the surgeon, and the second on his left-hand side.

Special instruments which I have found useful are Wilson's kidney pedicle clamp and a special gall-stone forceps made with a crocodile jaw beak, but otherwise having the same curve and size as a Desjardins forceps.

\section{The Operation of Cholecystectomy}

In no operation is the choice or the administration of the anaesthetic more important than in this. Perhaps it may be wise to pause for a moment and consider why the patient is being 
anaesthetised at all. It is for three main reasons: (I) that he, or she, shall not suffer immediate pain; (2) that pain impulses shall be blocked and so surgical shock lessened; and (3) that there shall be complete muscle relaxation, permitting the surgeon to work methodically and quickly without the use of retractors, thus again minimising surgical shock.

In gall bladder surgery beware of the "gas and oxygen apparatus" unless an artist in anaesthesia is in control, and remember that coroners have killed more patients than chloroform.

\section{The Position of the Patient.}

The patient lying supine on a horizontal table is so placed that the bridge is at the level of the tip of the ninth costal cartilage. The object of this bridge is to rotate the liver, and unless it is placed in exactly the right position it fails to achieve its purpose. It is quite impossible to see that the bridge is in exactly the right position unless all coverings from the abdomen are first removed. Care must be taken to see that the arms are not in contact with the side of the bridge: there has been at least one accident reported in which a carelessly placed arm was broken when the bridge was raised.

\section{Routes of Approach to the Gall Bladder.}

The three routes of approach to the gall bladder which are in general use are the right paramedial, the right transrectal, and the right subcostal.

The right paramedial incision, popularised by Moynihan, is probably the favourite incision, but it has very real disadvantages. It involves a separation of a great many of the rectus muscle fibres from their origin or insertion into the rectus muscle sheath at the level of the tendinous intersections. In this operation very careful dissection is required in freeing the rectus muscle from the sheath and a considerable amount of time is consumed. When the subsequent incision is made through the posterior sheath of the rectus a considerable thickness of transversalis muscle has to be cut through in the upper four inches of the wound, so that the claim of the adherents of this approach, that the muscle fibres are not damaged, is unfounded. The advantage of this operation is that the various incisions through the abdominal parietes are staggered. This again has the disadvantage that drainage through the original incision must keep the rectus out of place.

The transrectal incision, generally associated with Waring's name, is undoubtedly the quickest approach to the gall bladder. It also has the advantage that the preliminary exploratory incision may be relatively small and can be quickly extended. If the separation of the rectus fibres is made at the junction of the inner and middle thirds of the rectus, there is no danger of injuring the nerve supply to the muscle. Hernia following this incision is stated to be commoner than following either the paramedial or the subcostal incisions. That, in my opinion, is due to the fact that the incision in the muscle has been made too near the outer border. Drainage through the muscle for three to four days does not produce a tendency to post-operative hernia.

The subcostal incision described by Kocher is the easiest incision both to execute properly and to repair. The incision should be $\mathrm{I} \frac{1}{2}$ in. below the costal margin, and care must be taken to preserve the ninth intercostal nerve. This is undoubtedly the best incision in those cases in which there is any doubt as to whether the case will be "a difficult subject for anaesthesia."

Whichever route of approach is chosen, in very fat patients the incision through the skin should extend well above and below the projected incision through the muscle.

After the abdominal incision has been made the bridge of the table should be raised if this has not already been done.

\section{The Exposure of the Gall Bladder.}

The gall bladder and its ducts are exposed by displacing the stomach and the transverse colon downwards and further rotating the anterior border of the liver upwards.

Displacing the stomach and the transverse colon is done with a large abdominal pack or small towel maintained in position by the left hand of the first assistant. This pack is so often incorrectly placed that it may be worth while describing the procedure.

The lower border of the pack is introduced into the abdominal cavity anterior to the stomach and transverse colon, and just beneath the parietal peritoneum. The opposite border of the pack is passed above the stomach and transverse colon and beneath the peritoneum on each 
side of the wound. The surgeon passes his right hand over the pack until his fingers are lightly in contact with the posterior abdominal wall through the lesser omentum or to the right of it. The abdominal contents are then drawn gently downwards, the fingers still maintaining contact with the posterior abdominal wall. The left hand of the assistant is then placed above the surgeon's right hand and the surgeon's hand withdrawn, leaving the assistant with the fingers in contact with the posterior abdominal wall, a position which must be maintained until the moment has arrived for closure of the abdominal wall. With this position the use of retractors becomes unnecessary, and a most potent cause of surgical shock is avoided.

The gall bladder is now fully displayed by Moynihan's method of rotating the liver The fundus of the gall bladder, if not very tense, should be gripped with Wilson's kidney pedicle forceps. This forceps is held by a second assistant standing at the left of the surgeon.

The normal gall bladder is blue in colour and completely free from fat. The diseased gall bladder always contains a greater or less degree of fat in its sub-serous coat. In early stages of infection this fat is confined macroscopically to the immediate neighbourhood of the two lateral branches of the cystic artery.

If after his examination the surgeon be confirmed in his decision to remove the gall bladder, the question of exploration of the common hepatic duct has to be decided, and also the stage at which this exploration, if necessary, should take place.

The indications for exploration of the duct are all cases of cholelithiasis in which there is any possibility of a stone having been retained in the common bile duct. As it is almost impossible to exclude this possibility, the common bile duct should therefore always be explored unless there is a definite contra-indication. Much has been written and talked about methods for palpating the common bile duct, but all surgeons are agreed that it is impossible to determine positively by palpation that there is no stone in the duct. Negative results from palpation, therefore, should not deter the surgeon from opening the duct, whereas a positive result would only confirm his previous determination. It is obvious, therefore, that palpation of the duct is an unnecessary manoeuvre. Not only is it unnecessary, it is dangerous: it increases the time of the operation, it directly increases the patient's shock, and it is apt to lead to wrong conclusions because a slightly fibrotic pancreas or an enlarged lymph node may well be mistaken for a stone in the duct near the ampulla of Vater, and an unnecessarily prolongea search of the duct may be miade for the discovery of a non-existent stone.

In those cases in which the common bile duct has to be explored, the exploration should take place after the ligature and division of the cystic duct, and after ligature and division of the cystic artery and before the peritoneal reflection of the gall bladder has been incised. In this way a perfectly clear field, unhampered by escaping bile, is obtained for the two all-important ligatures of the cystic duct and artery, and the danger is avoided of inadvertently expressing a stone from the cystic duct into the common bile duct after the exploration.

\section{Adhesions.}

In almost every case adhesions to the gall bladder can be separated by swab dissection, and a full exposure of the gall bladder and its ducts obtained without the use of knife or scissors. Considerable patience is sometimes necessary. Dissections should always be started at the fundus of the gall bladder, and the adhesions should be peeled downwards towards their origin.

\section{Exposure and Ligature of the Cystic Duct.}

The cystic duct is identified as leaving the lower end of the gall bladder, but if its position is obscured by a .Hartmann's pouch, the pouch is seized with a curved gall bladder forceps and drawn upwards, the forceps being held by the second assistant. Blunt dissection immediately reveals the cystic duct which may be partially identified by its S-curve and by the lymph gland which invariably lies upon it; but the identity of the cystic duct can never be fully established until its connections at both ends have been properly explored. For this reason the cystic duct is dissected upwards until it is seen to expand into the gall bladder, and it is dissected downwards until the cystic duct, the common hepatic duct and the common bile duct are fully demonstrated at their meeting place. When all this has been done, the duct is ligatured with thread about $\frac{1}{4}$ in. from the common bile duct, its distal end is clamped with a Moynihan's gall bladder forceps, and the duct is divided between the ligature and the clamp. 
If the cystic artery is in its most usual place it will be found lying immediately behind क्The duct and now fully exposed. The artery should be traced to the gall bladder, and when fine surgeon has satisfied himself that there is no danger of ligaturing an aberrant right hepaic artery in addition to the cystic artery, the artery should be divided between ligatures of thread.

\section{Exploration of the Common Bile Duct.}

The common bile duct has already been exposed in order to identify the cystic duct. The duct should now be fixed with two catgut ligatures which are ultimately to be used to close duct. The ligatures should be made of 6-o catgut on an eyeless needle. The ligatures 震e passed through the anterior surface of the duct in its longitudinal axis, the points of entramce and emergence being about $\frac{\mathbf{3}}{16}$ in. apart. Each ligature is left long and held with a forceps, the left hand one by the first assistant and the right-hand one by the surgeon. The ligatures thezhselves are parallel and about $\frac{1}{8}$ in. from each other. When the needle penetrates the wall of the common bile duct a little bile escapes, this serving definitely to identify the common bile d $\overrightarrow{a c t}$ if further definition be necessary. The duct is carefully incised in a longitudinal direction agd midway between the two sutures, the opening in the duct extending slightly above and belaw them, thus giving a total length of about $\frac{3}{8}$ in. Bile which escapes is absorbed by packs previousty placed and effectively closing the foramen of Winslow. Gallstone forceps are introduced into the lower end of the common bile duct, and a careful search for stories is made. After exploring the lower end of the common bile duct the common hepatic duct is explored through the same opening. Finally the surgeon satisfies himself of the patency of the ampulla of Vater by passing a leaden probe through into the duodenum. Some surgeons like to use an aspirator, or even to irrigate the ducts. Both these practices are not without danger, and in my view are better avoided. Where there is any doubt of the completeness of the removal of any stones or "mud" that may be in the duct, it is better to establish wide drainage by passing a rubber tube of the calibre of a I6 English catheter into the opening in the duct and fixing it there with the two catgut sutues which have been used as retractors.

Normally the wound in the common bile duct is closed by tying loosely together the

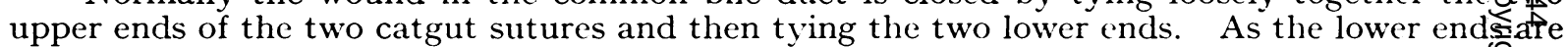
drawn together the knot in the upper ends will be drawn down and the edges of the cut approximated. Usually it is unnecessary to add any more stitches. It is true that the duct is not perfectly bile-tight, but it is both difficult and unnecessary to attempt to secure this, $\mathbf{a}$ all escape of bile from the duct will cease in about forty-eight hours, and in any event the leäk will be very slight, and drainage of the wound is being established for other reasons.

I make no attempt to cover the common bile duct with peritoneum except where the str $\overrightarrow{\mathbb{p}^{-}}-$ ping has been unusually wide, and then the edges are brought together loosely with one or two points of catgut.

\section{Removal of the Gall Bladder.}

The peritoneum on the medial side of the gall bladder is incised from below upwards, a a d then a similar incision is made through the peritoneum on the lateral side. The gall bladder then stripped from below upwards from its fossa until only the fundus remains attached to tBe anterior border of the liver. This serves as a useful retractor while the fossa for the gall bladder is dealt with. Care must be taken during separation of the gall bladder that the right hepagc duct is not damaged. As stated previously, this duct sometimes makes a loop behind the gadl bladder. Usually there is one fairly large vein in the fossa of the gall bladder that needs ligaturing. Very rarely an accessory bile duct is seen leaving the right lobe of the liver for the gasl bladder and requires ligaturing. More often than not, if such a duct exists it is unnoticed. Except in cases of acute infection, the fossa of the gall bladder may be closed by stitching together the peritoneal reflections on either side with three or four points of catgut. When this has beep satisfactorily accomplished, the gall bladder can be finally detached from the anterior border of the liver.

In many cases, particularly of old-standing gall bladder disease, it is not easy to get sufficie peritoneum to close the gall bladder fossa properly, although Maingot's method of injecting saline around the gall bladder at the line of the peritoneal reflection before incision may be successfully attempted if time permit. In such cases I usually obliterate the fossa by two sutures of well softened No. 4 catgut on a liver needle. Each suture is passed from the anterior surfa⿳⺈ 
of the liver straight through to the bordet of the fossa on its under surface, across the fossa, and then back through the liver substance to its anterior surface about $\frac{1}{2}$ in. from the point of entrance, and tied loosely. These two ligatures are quite sufficient to hold the edges of the fossa together and prevent haemorrhage. The very slight bleeding that occurs round the ligatures quickly stops. This method also has the advantage of speed in cases where this is of paramount importance.

All cases of cholecystectomy should be followed by drainage. This is best performed through a stab incision to the lateral side of the vertical abdominal incision or below the subcostal incision. The drainage tube should be placed so that its end is resting immediately to the right of the common bile duct and touching the posterior abdominal wall. In the last stage of the operation, when the skin is being closed, the drainage tube should be carefully withdrawn for $I \frac{1}{2}$ in. so that it can be now quite certain that not only is it not resting on the posterior abdominal wall or the common bile duct, but also that it has not been brought too far forward to act as an effective drain. A badly placed drainage tube is one of the most common sources of pain and inhibition of lung function in the immediate post-operative stage.

\section{Cholecystectomy in Jaundiced Patients}

\section{Choice of Anaesthetic.}

In all cases of jaundice and impaired liver function the use of chloroform is contra-indica ted

The best anaesthetics are Cyclopropane or a high spinal anaesthetic coupled with an intravenous adrenalin drip by the Frankis Evans technique. (See p. 56.)

The incision of choice is the transrectal (Waring's), because it is the quickest and because there is much less haemorrhage with this than with either the paramedial or the subcostal.

If jaundice has persisted for several weeks before operation, and if the patient's condition be poor, it is wise to explore the common bile duct before dividing the cystic duct. If the common bile duct is found to contain white bile, the duct should be drained and the removal of the gall bladder deferred until the patient's liver function has been restored and jaundice lost.

\section{Cholecystostomy}

This operation is seldom performed, and has long since been abandoned as a cure for gall stones. It may occasionally be performed in cases of empyaemia of the gall bladder, or in low obstructive jaundice, in which the patient's condition is too bad to permit of more radical surgery. It is sometimes recommended in the treatment of acute pancreatitis, but in my opinion this is unsound, because the arguments for its use have been based upon wrong premises, and it does, in fact, prove harmful.

There is some evidence to show that acute haemorrhage pancreatitis may be due to infection, and that the infection is derived from the gall bladder. It has therefore been argued that the gall bladder should be drained; but the exudate in the acute stage of pancreatitis is invariably sterile, although a haemolytic streptococcus may occasionally be cultivated from tissues in the neighbourhood of the pancreas.

Wilkie has shown that when a haemolytic streptococcus attacks the gall bladder it is in the wall, not the lumen, and a great deal of work has been done-to mention only that of Maugeret-to show that infection passes from the gall bladder to the pancreas via the lymphatics.

Again, there is no evidence to show that bile regurgitates down the main pancreatic duct during life, and thus carries "infected" bile into the pancreatic tissue.

Finally, the patient cannot afford to be deprived of bile. In spite of the fact that sugar metabolism is seldom affected by acute pancreatitis, patients waste very rapidly. One of the causes of this wasting is loss of the pancreatic lipolitic ferment. The withdrawal of bile from the digestive circulation by the operation of cholecystostomy still further depletes the patient's power of fat digestion.

\section{Operation.}

After opening the abdomen the gall bladder area should be carefully packed off, and if the gall bladder is tightly distended it should be aspirated. The fundus of the gall bladder is picked up with two fine Kocher forceps placed $\frac{1}{2}$ in. apart, and in such a position that the nearer is a 
full inch from the anterior border of the liver. A "purse-string" suture of $O$ catgut is introduced around the forceps, but is not tied, and the needle is left attached. The walls of the gall bladder are then incised between the forceps, care being taken to see that the mucous membrane is divided and not pushed in front of the knife or scissors. If there is any doubt, the mucous membrane should be picked up with another forceps. A No. I6 red rubber English catheter is introduced through the opening in the gall bladder projecting rather less than half way towards the neck. The needle of the "purse-string" suture is now passed through the tube and the suture tied. A second "purse-string" suture is introduced about half an inch from the first, and the first suture inverted in the "ink-bottle" manner and buried by tying the second suture. The second suture must not penetrate the tube as this may allow a leak of bile into the peritoneum Next a stab incision is made through the abdominal wall about $I \frac{1}{2}$ in. below the tip of the ninth costal cartilage. The free end of the tube is brought out through the opening and is fixed to the skin with a silkworm gut suture. The main abdominal wall is closed without drainage.

\section{Cholecystotomy}

Cholecystotomy is not a surgical operation. One case may be worth recording because of several interesting features. An officer in the last war was shot through the right lung by a round lead shrapnel ball. The bullet was located by X-ray in the substance of the liver, and no attempt was made to remove it. After a B. pyocyaneus empyaemia of the right pleura had subsided his convalescence was delayed by repeated attacks of biliary colic. I eventually operated and found that the shrapnel ball was in the gall bladder, which it had apparently entered through the fossa for the gall bladder. I opened the viscus, which appeared to be perfectly healthy, removed the bullet, and closed the gall bladder with two layers of sutures. The patient made an uninterrupted recovery. But if I were confronted with the same'problem again I should certainly remove the gall bladder.

\section{Cholecystgastrostomy and Cholecystenterostomy}

These operations are performed for jaundice due to permanent obstruction of the common bile duct below the entrance of the cystic duct. The commonest causes of the obstruction are carcinoma of the head of the pancreas and carcinoma arising in the ampulla of Vater or in the common bile duct proper.

Almost anything is better than the death of the deeply jaundiced patient with his intense mental depression and intolerable skin irritation. The operation, therefore, must be hazarded in most cases, but no unnecessary risk should be run. Therefore it is necessary to choose the speediest incision and the simplest manœuvres with the minimum amount of handling of intraperitoneal organs. For these reasons I prefer cholecystgastrostomy to cholecystenterostomy, whether with the first part of the jejunum or the duodenum. When the operation is performed, not only is the gall bladder grossly distended, but the liver also is considerably swollen; and it must be remembered that if the operation has been successful the fundus of the gall bladder will tend to be retracted several inches upwards towards or beneath the right costal margin, dragging with it the viscus to which it has been anastomosed. For this reason a point as near the lesser curvature of the stomach and as near the pylorus as is conveniently possible should be taken. The opening in the stomach should be longitudinal as opposed to the transverse opening of posterior gastroenterostomy. The technique of anastomosis does not need description: suffice it to say it is done with the use of clamps after aspiration of the gall bladder.

I have never seen any untoward results from the presence of bile flowing direct from the gall bladder into the stomach, but I have seen a case of vomiting following anastomosis of the much dilated gall bladder high up on the greater curvature of the stomach, but I think this was due to, the mechanics of the operation and not to the bile.

Where cholecystenterostomy is preferred to cholecystgastrostomy, a loop of jejunum may be brought up in front of the transverse colon and anastomosed to the gall bladder. This has obvious mechanical disadvantages, and so has the operation of bringing the loop through the gastrocholic omentum. However, in some cases it is not easy to effect an anastomosis between the gall bladder and the stomach, and in those cases cholecysto-jejunostomy is the operation I would choose, and the simpler the operation of choice the more likely its success. The operation after all is a palliative one, and the surgeon is primarly concerned with giving the patient 
a few months of comfort rather than years of useful work. If he bear this in mind he will realise that the expectation of life of the patient is directly proportionate to the speed and simplicity of his operation.

\title{
THE DOME
}

"Wisdom is the principal thing; therefore get wisdom: and with all thy getting get understanding."-Proverbs iv. 7.

\section{ANAESTHESIA IN GALL BLADDER SURGERY}

\author{
By FRANKIS EVANS, M.B., B.S., D.A. \\ (Anaesthetist, St. Bartholomew's Hospital; St. Mark's Hospital, etc.)
}

Surgery of the gall bladder necessitates the closest co-operation between the surgeon and the anaesthetist. The former has a delicate operation to perform, and is often working in the depths of a fat abdomen. The anaesthetist has to deal with a patient who is only too frequently fat and portly and difficult to anaesthetise. Even as the accompanist can make or mar the vocalist's performance, so can a bad anaesthetic render the surgeon's task well nigh impossible. The anaesthetist has a threefold duty, firstly to the patient, secondly to the surgeon, and lastly to himself. The patient should be anaesthetised with as little disturbance and unpleasantness as possible, and by that method which interferes least with the physiological processes of the body. The respiration should resemble that of sleep, so that the body does as little work as possible, and the surgeon's task is aided by the diminished movement. The surgeon needs absolute muscular relaxation of the abdomen as well as quiet respiration. Lord Moynihan's dictum, "Every pull means a pain," is very true, and if relaxation is complete no retractors need be used, and the time taken over the operation is materially reduced. The longer a patient is on the operating table the more likely is he to suffer with post-operative complications. The anaesthetist's duty to himself is to know his own limitations, and not to attempt that technique with which he is not really familiar, for this leads to failure and even to disaster. Ether may be undesirable and unpleasant post-operatively from all points of view, but it is better to be sick and to cough than to be the subject of an inquest! A sane and balanced outlook is essential.

The choice of anaesthetic and the method of administration is dictated by the condition of the patient, the time the operation will take and one's own personal prejudice. Inhalation anaesthesia held pride of place for many years, and both chloroform and ether have had their protagonists. The necessary muscular relaxation can be obtained in other ways, however, and the modern anaesthetist has a choice of several methods. Intravenous anaesthesia, spinal analgesia, and regional analgesia can each be used to give relaxation, but care must be exercised in employing any one of these methods. The indications and contra-indications for their use are discussed below. The patient's condition, both mental and physical, must be carefully considered. The frightened patient is more difficult to anaesthetise than the placid one, and requires more anaesthetic. The "Falstaffs" are also difficult, largely owing to their build. On the other hand these portly patients who habitually do themselves well seldom suffer with post-anaesthetic vomiting! The anaesthetist should know the condition of the patient's cardiovascular system (myocardium, blood pressure, arterio-sclerosis, etc.). The liver and renal function, too, should be known, for the blood urea is a very good guide of a patient's general condition. It is obvious that a fat, jaundiced patient with a flabby heart and poor excretory system will need more care than will a young, thin, wiry patient. Not only has the anaesthetist several separate methods of approach, but he may use a combination of methods.

Inhalation anaesthesia for gall bladder surgery calls for third plane anaesthesia, that is to say, full muscular relaxation. The surgeon is completely in the hands of his anaesthetist when inhalation anaesthesia alone is used, for if the patient be not sufficiently relaxed, the operation may be well nigh impossible. Chloroform alone can be used, but is not recommended, and 